\title{
Determination of volatile components in fermented soybean prepared by a co- culture of Bacillus subtilis and Rhizopus oligosporus
}

\author{
*Chukeatirote, E., Eungwanichayapant, P.D. and Kanghae A. \\ School of Science, Mae Fah Luang University, Chiang Rai 57100, Thailand
}

\begin{abstract}
Article history:
Received: 22 June 2017

Received in revised form: 15 July 2017

Accepted: 18 July 2017

Available Online: 19 July 2017
\end{abstract}

Keywords:

Bacillus subtilis,

Fermented soybean,

Rhizopus oligosporus,

Volatile components

DOI:

http://doi.org/10.26656/ fr.2017.6.066

\begin{abstract}
Fermented soybeans are traditional foods widely consumed in many countries (i.e., Natto in Japan, Jang in Korea, Kinema in India, Douichi in China, and Thua Nao in Thailand). In this study, an attempt was made with an expectation to improve the fermentation process using a co-culture of Bacillus subtilis and Rhizopus oligosporus. Initially, the raw soybeans were washed, sterilized by autoclaving, and inoculated with two inocula; for this, three different ratios between B. subtilis and R. oligosporus used were 100:0, 50:50, and $0: 100$. The fermentation was then carried out at $30^{\circ} \mathrm{C}$ for 3 days. The volatile compounds in the non-fermented and the fermented soybean products were determined by gas chromatography/ mass spectrometry (GC/MS) and extracted using a solid phase microextraction (SPME) technique. In total, 165 compounds were identified in the nonfermented and the fermented soybean products. For the non-fermented products, the predominant volatile compounds were alcohols (25.81\%), aldehydes (13.64\%), acids and esters $(7.57 \%)$, furans $(6.13 \%)$ and ketones $(0.88 \%)$. In contrast, the major volatiles compounds presented in the fermented soybeans were as follows: i) The treatment of 100:0 consisted of acids and esters (35.89\%), alcohols (14.55\%), aldehydes (8.72\%), ketones (4.97\%), pyrazines (4.87\%), and furans (4.22\%); ii) 50:50 comprised of acids and esters $(55.62 \%)$, alcohols $(16.22 \%)$, aldehydes $(7.80 \%)$, pyrazine $(3.65 \%)$, ketones (2.55\%), furans (1.67\%), and aromatic compounds (1.46\%); and iii) 0:100 included acids and esters $(66.50 \%)$, alcohols (15.44\%), aldehydes $(2.59 \%)$, ketones $(2.72 \%)$, furans $(1.89 \%)$, aromatic compounds $(1.80 \%)$, pyrazines $(1.35 \%)$, and sulphur containing compounds $(0.24 \%)$ respectively.
\end{abstract}

\section{Introduction}

Fermented soybean is one of the traditional foods typically consumed as a condiment or a flavouring agent in Korea (Chongkukjang), Japan (Natto), Thailand (Thua Nao), India and Nepal (Kinema) (Hui and Evranuz, 2012). These products are similar, typically made from cooked soybeans (either by steaming or boiling), and naturally fermented. Interestingly, Bacillus species are found to be predominant and thus are considered to be responsible for these fermentation processes (Steinkraus, 2002).

Fermented soybean products are unique in terms of their appearance, texture, taste, and odour. These characteristics are obtained as a result of hydrolysis, biochemical metabolism, and microbial activity. In general, most fermented soybean products possess ammonia-like and fishy odours; such features are mainly derived from the hydrolysis of soy proteins (protein degradation into peptides, amino acids, and ammonia). As a result, this "spoiled" odour is often regarded as "unpleasant" attribute and hence restricted their use to some certain groups of consumers. Natto, in particular, is different; the Natto product is commercially produced by using a pure starter culture of Bacillus subtilis strain natto (Kiuchi and Watanabe, 2004). The odour of the Natto product is mild and often recognised as "nattolike" odour (or fruity/nutty aroma) which indicates a good quality of the fermented Natto product.

Consequently, quantitative determination of volatile compounds in the fermented soybean products is of great importance. Considered that olfactory sensation is one of the prime attributes affecting the consumer's acceptability, it is therefore not surprising that many 
researchers have paid their attention to work on this issue. Numerous reports have described the volatile components in the fermented soybean products; these include Korean Chungkukjang (Tanaka et al., 1998), Natto (Sugawara et al., 1998), and Thua Nao (Leejeerajumnean et al., 2001; Dajanta et al., 2011). Although these studies have shown that the frequent compounds in the fermented soybean products include esters, acids, pyrazines, and phenolic compounds, it should be noted that there are variations of the volatile component profiles among these fermented soybeans mainly depending on the soybean variety, microflora involved, and the processing conditions (Tanaka et al., 1998; Leejeerajumnean et al., 2001).

As mentioned, it is suggested that the bacterial members belonging to the Genus Bacillus are responsible for the fermentation of these soybean products. In this study, an attempt was made with an expectation to improve the fermentation process using a co-culture between the bacterium Bacillus subtilis and the fungus Rhizopus oligosporus. The major purpose of this present study was to determine the volatile component profiles of the developed products.

\section{Materials and methods}

\subsection{Microbial strains and culture condition}

Bacillus subtilis strain TN51 previously isolated (Dajanta et al., 2009) was used as a bacterial starter culture. For inoculum preparation, the bacterial strains were grown in nutrient broth at $37^{\circ} \mathrm{C}$ for $24 \mathrm{~h}$. The cells were then harvested, resuspended in sterile distilled water and adjusted to $10^{4} \mathrm{CFU} / \mathrm{mL}$. The fungus Rhizopus oligosporus NRRL 5905 was obtained from the Agricultural Research Service Culture Collection, Bacterial Foodborne Pathogens and Mycology Research Unit National Center for Agricultural Utilization Research (USA). To prepare as a starter culture, the fungus was cultured on potato dextrose agar at $30^{\circ} \mathrm{C}$ for $3-4$ days. The spores were then harvested, resuspended in sterile distilled water and adjusted to $10^{4}$ spores $/ \mathrm{ml}$.

\subsection{Soybean fermentation}

Soybeans were washed and soaked in water for $16 \mathrm{~h}$ at room temperature. Prior to fermentation, two different methods were employed to prepare cooked nonfermented soybeans (CNF). For the first batch, soaked soybeans were cooked by boiling for $3 \mathrm{~h}(\mathrm{CNF} 1)$ and left to ferment by naturally occurring microbes at $30^{\circ} \mathrm{C}$ for $72 \mathrm{~h}$ (TNM). For another batch, the soaked soybeans were autoclaved at $121^{\circ} \mathrm{C}$ for $40 \mathrm{~min}(\mathrm{CNF} 2)$, and the fermentation was then carried out using the microbial inocula $\left(10^{4} \mathrm{CFU}\right.$ (or conidia) per $\mathrm{g}$ of the autoclaved soybeans). Three treatments with different ratios between $B$. subtilis and $R$. oligosporus were prepared as follows: i) 100:0 (TNB), ii) 50:50 (TNBR), and iii) 0:100 (TNR). After that, the soybeans were incubated at $30^{\circ} \mathrm{C}$ for $72 \mathrm{~h}$.

\subsection{Volatile composition analysis}

Fifty gram of fermented soybean paste sample was placed into a $125 \mathrm{~mL}$ glass bottle and covered with a cap. In this study, a solid phase microextraction (SPME) device (Bellefonte, PA, USA) with a 50/30 $\mu \mathrm{m}$ divinylbenzene-carboxen-polydimethylsiloxane (DVBCAR-PDMS) fibre was used for the extraction of volatile compounds. The bottle containing sample was left at $60^{\circ}$ $\mathrm{C}$ in a water bath for $30 \mathrm{~min}$ to equilibrate. Subsequently, the SPME fibre was inserted into the headspace above the soybean sample for adsorption of the headspace volatiles for $30 \mathrm{~min}$ (Qin and Ding, 2007). After extraction, the loaded SPME fibre was immediately removed from the sample vial and inserted into the injection port of a gas chromatograph (GC) for further analysis. Two replicates extractions were done for each sample.

The volatile compounds of the extracts were subsequently determined using GC/MS. The system was comprised of the Agilent $6890 \mathrm{~N}$ gas chromatograph and the Agilent $5973 \mathrm{~N}$ mass spectrometer (Agilent Technologies, USA). Helium gas, $1.0 \mathrm{~mL} / \mathrm{min}$, was passed through the Agilent HP-5MS capillary column (30m $\times 0.25 \mathrm{~mm}$ ID, $0.25 \mu \mathrm{m}$ in film thickness). The volatiles was desorbed from the SPME fibre by heating at $250{ }^{\circ} \mathrm{C}$ for $5 \mathrm{~min}$., then held at $40^{\circ} \mathrm{C}$ for $3 \mathrm{~min}$, heated to $230{ }^{\circ} \mathrm{C}$ for $6 \mathrm{~min}$, and held at $230{ }^{\circ} \mathrm{C}$ for a further 10 min. The mass spectrophotometer was operated in the electron impact mode with ion source temperature of 230 ${ }^{\circ} \mathrm{C}$, using an ionisation voltage of $70 \mathrm{eV}$, and the mass range was $30-450 \mathrm{amu}$. The compounds were then identified by comparison with mass spectra from the library database (NIST14).

\section{Results and discussion}

Table 1 shows proximate profiles of the volatile compounds in cooked non-fermented soybeans (CNF1 and CNF2) and fermented soybeans (TNM, TNB, TNR, and TNBR). In general, there was a shift in the volatiles determined depending on the cooking process as well as the fermenting microbes. For comparative purpose in terms of the volatile types and amounts, the CNF1 
Table 1. Volatile compounds in cooked non-fermented soybeans and fermented soybeans

\begin{tabular}{|c|c|c|c|c|c|c|}
\hline Volatile compounds & CNF1 & CNF2 & TNM & TNB & TNR & TNBR \\
\hline \multicolumn{7}{|l|}{ Alcohols } \\
\hline Ethanol & - & $5.72 \pm 0.00$ & $0.20 \pm 0.11$ & $4.11 \pm 0.68$ & $5.66 \pm 0.42$ & $2.33 \pm 0.00$ \\
\hline 4-Penten-2-ol & $1.49 \pm 0.52$ & - & - & - & $1.27 \pm 0.18$ & - \\
\hline Dimethyl-silanediol & - & $3.54 \pm 0.08$ & - & - & $4.53 \pm 1.86$ & $4.02 \pm 0.02$ \\
\hline 3-Methyl-2-butanol & - & - & - & $0.54 \pm 0.12$ & - & - \\
\hline 3-Methyl-1-butanol & - & - & - & - & $0.50 \pm 0.24$ & $0.34 \pm 0.01$ \\
\hline 2,3-Butanediol & - & - & $7.23 \pm 0.08$ & $3.23 \pm 0.26$ & $0.53 \pm 0.06$ & $2.93 \pm 0.02$ \\
\hline 2,5-Dimethyl-3-hexanol & - & - & - & $0.39 \pm 0.08$ & - & - \\
\hline 2,6-Dimethyl-4-heptanol & - & $0.53 \pm 0.15$ & - & - & - & $0.42 \pm 0.43$ \\
\hline 1-Octen-3-ol & $0.60 \pm 0.62$ & $8.99 \pm 0.39$ & $0.46 \pm 0.01$ & $1.65 \pm 0.31$ & $0.78 \pm 0.02$ & $0.84 \pm 0.02$ \\
\hline Benzyl alcohol & - & - & $0.22 \pm 0.00$ & $0.41 \pm 0.10$ & $0.17 \pm 0.01$ & $0.73 \pm 0.01$ \\
\hline 1-Octanol & - & - & - & $0.24 \pm 0.10$ & - & - \\
\hline 2-Cyclohexen-1-ol & $0.62 \pm 0.00$ & - & - & $0.25 \pm 0.05$ & - & $0.18 \pm 0.00$ \\
\hline 2-Octen-1-ol & - & - & - & - & - & $0.31 \pm 0.00$ \\
\hline 1-Dodecanol & - & - & - & $0.27 \pm 0.02$ & - & - \\
\hline 2-Methylene cyclopentanol & - & - & - & - & $0.11 \pm 0.01$ & - \\
\hline Phenyl ethyl Alcohol & - & - & - & - & $1.90 \pm 0.74$ & $3.15 \pm 0.01$ \\
\hline 3,4-Dimethylbenzyl alcohol & - & - & - & - & - & $0.13 \pm 0.01$ \\
\hline 3-Ethyl-3-octanol & - & - & - & - & - & $0.10 \pm 0.00$ \\
\hline (R)-(-)-14-Methyl-8-hexadecyn-1-ol & - & - & - & - & - & $0.06 \pm 0.00$ \\
\hline $\begin{array}{l}\text { 2,5-Bis(1,1-dimethylethyl) } 1,4 \text { - } \\
\text { benzenediol }\end{array}$ & - & - & - & - & - & $0.16 \pm 0.00$ \\
\hline Total Alcohols (20) & $2.71 \pm 1.14$ & $18.78 \pm 0.62$ & $8.11 \pm 0.20$ & $11.09 \pm 1.72$ & $15.45 \pm 3.54$ & $15.70 \pm 0.53$ \\
\hline \multicolumn{7}{|l|}{ Aldehydes } \\
\hline Methional & - & - & - & - & - & $0.22 \pm 0.01$ \\
\hline 3-Methyl-butanal & - & - & - & - & - & $0.63 \pm 0.02$ \\
\hline Acetaldehyde & - & - & $0.53 \pm 0.06$ & $0.58 \pm 0.19$ & $0.15 \pm 0.01$ & $0.32 \pm 0.00$ \\
\hline (Z)-Heptenal & - & $0.89 \pm 0.11$ & - & - & $0.13 \pm 0.01$ & - \\
\hline (Z)-7-Hexadecenal & $0.21 \pm 0.00$ & - & - & - & - & - \\
\hline Hexanal & - & - & - & - & - & $0.17 \pm 0.03$ \\
\hline Benzaldehyde & $0.28 \pm 0.06$ & $6.46 \pm 0.33$ & $7.29 \pm 0.72$ & $3.24 \pm 0.57$ & $1.03 \pm 0.04$ & $1.55 \pm 1.14$ \\
\hline Benzeneacetaldehyde & - & $1.31 \pm 0.02$ & $1.02 \pm 0.06$ & $1.78 \pm 0.19$ & $0.74 \pm 0.06$ & $2.04 \pm 0.04$ \\
\hline 14-Heptadecenal & $0.34 \pm 0.06$ & - & - & - & - & - \\
\hline (E,E) 2,4-Haptadienal & - & - & - & - & $0.17 \pm 0.02$ & - \\
\hline Nonanal & - & $0.92 \pm 0.08$ & $0.39 \pm 0.22$ & $0.29 \pm 0.08$ & $0.16 \pm 0.01$ & $1.01 \pm 0.00$ \\
\hline Piperonal & - & - & - & $0.28 \pm 0.06$ & - & - \\
\hline (E)-2-Nonenal & - & - & - & - & - & $0.8 \pm 0.01$ \\
\hline Decanal & - & - & $0.14 \pm 0.02$ & - & - & $0.15 \pm 0.01$ \\
\hline Alpha-ethylidene-benzeneacetaldehyde & - & - & $0.18 \pm 0.04$ & $0.48 \pm 0.08$ & $0.21 \pm 0.03$ & $0.66 \pm 0.69$ \\
\hline Total Aldehydes (15) & $0.83 \pm 0.12$ & $9.92 \pm 0.54$ & $9.55 \pm 1.12$ & $6.65 \pm 1.17$ & $2.59 \pm 0.18$ & $7.55 \pm 1.95$ \\
\hline \multicolumn{7}{|l|}{ Aromatic compounds } \\
\hline 1,3-Diethenyl-benzene & - & - & - & - & - & $0.25 \pm 0.02$ \\
\hline 1-Pentenyl-benzene & - & - & - & - & - & $0.21 \pm 0.01$ \\
\hline 3,4-Dimethyl benzenamine & - & - & - & - & - & $0.72 \pm 0.01$ \\
\hline m-Phenethyl-benzonitrile & - & - & - & - & - & $0.23 \pm 0.00$ \\
\hline 3-Methoxybenzylamine & - & - & - & - & $0.15 \pm 0.00$ & - \\
\hline 4-(Nonafluoro-tert-butyl) nitrobenzene & - & - & - & $0.17 \pm 0.04$ & - & - \\
\hline 3-Methoxybenzylamine & - & - & - & - & $0.15 \pm 0.00$ & - \\
\hline 2-Nitro-N-phenyl-benzenesulfonamide & - & - & - & - & $1.65 \pm 0.01$ & - \\
\hline
\end{tabular}


Table 1. (Continue)

\begin{tabular}{|c|c|c|c|c|c|c|}
\hline Volatile compounds & CNF1 & CNF2 & TNM & TNB & TNR & TNBR \\
\hline Ethenyloxybenzene & - & - & $1.02 \pm 0.00$ & - & - & - \\
\hline Total Aromatic compounds (8) & $0.00 \pm 0.00$ & $0.00 \pm 0.00$ & $1.02 \pm 0.00$ & $0.17 \pm 0.04$ & $1.80 \pm 0.01$ & $1.41 \pm 0.04$ \\
\hline \multicolumn{7}{|l|}{ Ketones } \\
\hline 2-Pentanone & $4.11 \pm 0.68$ & - & - & $1.21 \pm 0.01$ & $0.31 \pm 0.00$ & $1.22 \pm 0.02$ \\
\hline 3-Penten-2-one & - & - & - & $0.41 \pm 0.27$ & $0.39 \pm 0.04$ & - \\
\hline 2-Pentadecanone & $0.88 \pm 0.02$ & - & - & - & - & - \\
\hline 1-Methoxy-2-propanone & - & - & - & - & $0.51 \pm 0.01$ & - \\
\hline 2-Heptanone & $0.42 \pm 0.01$ & - & $0.25 \pm 0.01$ & $0.29 \pm 0.04$ & - & - \\
\hline Pyrovalerone & - & - & - & $0.18 \pm 0.13$ & - & - \\
\hline 1,3-Diphenyl2-propanone & - & - & - & $0.22 \pm 0.0$ & - & \\
\hline 2-Ethyl-cyclobutanone & - & - & - & - & - & $0.62 \pm 0.04$ \\
\hline 2,5-Dimethyl-3-hexanone & - & - & - & - & - & $0.37 \pm 0.33$ \\
\hline 5-Hydroxy-3,6-dimethyl4-octanone & - & - & - & - & - & $0.44 \pm 0.04$ \\
\hline 2,3-Octanedione & - & - & - & - & - & $0.14 \pm 0.01$ \\
\hline 2,3-Butandione & - & - & $0.06 \pm 0.04$ & $1.30 \pm 0.78$ & $0.06 \pm 0.01$ & - \\
\hline 2,6-Dihydroxyacetophenone & - & - & - & $0.18 \pm 0.02$ & - & - \\
\hline 3-Octanone & - & - & - & - & - & $0.23 \pm 0.00$ \\
\hline 2-Ethyl-cyclohexanone & - & - & - & - & - & $0.22 \pm 0.02$ \\
\hline Acetoin & $4.16 \pm 0.68$ & $0.64 \pm 0.02$ & $0.25 \pm 0.01$ & & $1.29 \pm 0.84$ & $0.45 \pm 0.03$ \\
\hline 2-Bromo-1-phenyl-1-propanone & - & - & - & - & $0.16 \pm 0.00$ & - \\
\hline 2-Undecanone & - & - & $0.37 \pm 0.02$ & - & - & - \\
\hline 2-Tridecanone & - & - & $0.58 \pm 0.06$ & - & - & - \\
\hline Total Ketones (19) & $9.57 \pm 1.39$ & $0.64 \pm 0.02$ & $1.51 \pm 0.14$ & $3.79 \pm 1.27$ & $2.72 \pm 0.91$ & $2.47 \pm 0.47$ \\
\hline \multicolumn{7}{|l|}{ Acids and esters } \\
\hline Acetic acid & $0.98 \pm 0.68$ & - & $2.53 \pm 0.01$ & - & $0.51 \pm 0.08$ & $1.23 \pm 0.02$ \\
\hline 3-Methyl-hexanoic acid & - & - & - & - & - & $0.57 \pm 0.00$ \\
\hline 3-Methyl-butanoic acid & - & - & $0.44 \pm 0.06$ & $0.43 \pm 0.08$ & - & $0.26 \pm 0.01$ \\
\hline 2-Methyl-propanic acid & - & - & - & $0.30 \pm 0.04$ & - & - \\
\hline 3-Methyl-pentanoic acid & - & - & - & $0.15 \pm 0.01$ & - & - \\
\hline 2-Methyl-hexanoic acid & - & - & $0.14 \pm 0.01$ & $0.39 \pm 0.03$ & - & - \\
\hline 2-Methyl-decanoic acid & - & - & - & $0.25 \pm 0.01$ & - & - \\
\hline 2-Methyl-butanoic acid & - & - & $0.56 \pm 0.03$ & - & - & $0.38 \pm 0.01$ \\
\hline Tetradecanoic acid & - & - & - & - & - & $0.06 \pm 0.01$ \\
\hline Octanoic acid & $13.18 \pm 0.02$ & - & - & $0.34 \pm 0.06$ & - & - \\
\hline n-Hexadecanoic acid & $0.19 \pm 0.00$ & $0.41 \pm 0.01$ & $0.33 \pm 0.47$ & $7.12 \pm 0.05$ & $0.61 \pm 0.16$ & $0.68 \pm 0.00$ \\
\hline Oleic acid & $0.26 \pm 0.00$ & - & - & $1.32 \pm 0.02$ & - & - \\
\hline Octadecanoic acid & $20.50 \pm 0.00$ & - & - & $0.57 \pm 0.02$ & - & - \\
\hline $\begin{array}{l}2,6 \text {-Dihydroxybenzoic acid, } 3 \text { TMS de- } \\
\text { rivative }\end{array}$ & - & - & - & $1.06 \pm 0.06$ & $0.18 \pm 0.00$ & - \\
\hline 6-Octadecanoic acid & - & - & - & $0.06 \pm 0.00$ & - & - \\
\hline 1-Butanol, 3-methyl-acetate & - & - & - & - & $0.24 \pm 0.01$ & - \\
\hline Propanoic acid, 3-methyl-,methyl ester & - & - & - & - & $0.21 \pm 0.01$ & $0.14 \pm 0.01$ \\
\hline Formic acid, 1-methylpropyl ester & - & - & - & $0.45 \pm 0.00$ & - & - \\
\hline $\begin{array}{l}\text { Hexanoic acid, 2,4-dimethyl-methyl } \\
\text { ester }\end{array}$ & - & - & - & $0.61 \pm 0.28$ & - & - \\
\hline Butanoic acid, 3-hydroxy-ethyl ester & - & - & - & $2.60 \pm 0.66$ & - & $0.22 \pm 0.12$ \\
\hline $\begin{array}{l}\text { Butanoic acid, 3-(phenylamino)-ethyl } \\
\text { ester }\end{array}$ & - & - & - & - & $0.05 \pm 0.00$ & - \\
\hline $\begin{array}{l}\text { Butanoic acid, 2-methyl 2-methylpropyl } \\
\text { ester }\end{array}$ & - & - & - & - & - & $0.25 \pm 0.01$ \\
\hline
\end{tabular}


Table 1. (Continue)

\section{Volatile compounds}

Benzoic acid, methyl ester

1-Octyl trifluoroacetate

Formic acid, 2,6-dimethoxyphenyl ester

Ethyl tridecanoate

2-Propenoic acid, 3-phenyl-, methyl ester

Heptanoic acid, 2-propyl- methyl ester

2,5-Dichloro-3-nitrobenzoic acid

Octanoic acid ethyl ester

Benzeneacetic acid, ethyl ester

Tetradecanoic acid, ethyl ester

Pentadecanoic acid, ethyl ester

Hexadecanoic acid, methyl ester

Ethyl 9-hexadecenoate

Benzeneacetic acid, .alpha.,3,4-tris [(trimethylsilyl)oxy]-, methyl ester Hexadecanoic acid, ethyl ester

Dodecanoic acid, ethyl ester

9,12-Octadecadienoic acid methyl ester

(E)-9-Octadecadienoic acid methyl ester Octadecanoic acid

(Z,Z,Z) 9,12,15-Octadecatrienoic acid

11-Octadecenoic acid, methyl ester

13-Octadecenoic acid, methyl ester

Stearic acid methyl ester

Linoleic acid ethyl ester

Oleic acid ethyl ester

(Z,Z)-9,12-Octadecadienoic acid methyl ester

(E)-9-Octadecenoic acid ethyl ester

Octadecanoic acid, ethyl ester

9-cis,11-trans-Octadecadienoate

9,12-Octadecadienoic acid ethyl ester

Butyl-9,12-octadecadienoate

Methyl-9,12-heptadecadienoate

\section{CNF1}

CNF2

TNM

TNB

$0.40 \pm 0.0$

TNBR

$\begin{array}{ll}- & - \\ - & - \\ - & - \\ - & - \\ - & -\end{array}$

$0.16 \pm 0.00$

$1.36 \pm 0.01$

$0.76 \pm 0.01$

$0.06 \pm 0.01$

$0.12 \pm 0.02$

$0.66 \pm 0.02$

$1.08 \pm 1.12$

$0.29 \pm 0.01$

$0.54 \pm 0.17$

$0.22 \pm 0.00$

$$
1.19 \pm 0.08
$$

$0.17 \pm 0.04$

$9.88 \pm 0.01$

$13.24 \pm 0.53$

$10.61 \pm 0.04$

$0.12 \pm 0.01$

$0.16 \pm 0.01$

$1.26 \pm 0.06$

$1.53 \pm 1.24$

$0.77 \pm 0.04$

$4.11 \pm 0.00$

$1.12 \pm 0.04$

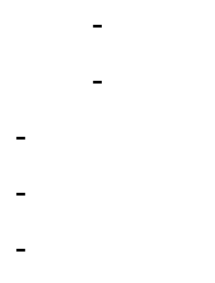

$0.27 \pm 0.00$

$2.38 \pm 0.04$

$0.16 \pm 0.01$

$25.89 \pm 0.48$

$1.48 \pm 0.00$

$0.47 \pm 0.00$

$0.32 \pm 0.00$

$13.71 \pm 0.28$

$16.69 \pm 0.25$

$9.44 \pm 0.00$

$0.16 \pm 0.01$

$7.48 \pm 0.00$

$\begin{array}{ll}- & 1.53 \pm 0.08\end{array}$

-

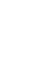
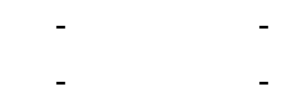

$1.78 \pm 0.03$

$1.25 \pm 0.00$

$1.25 \pm 0.25$

$1.25 \pm 0.00$

$0.38 \pm 0.10$

$0.34 \pm 0.00$

$0.61 \pm 0.00$

$0.84 \pm 0.01$

$0.20 \pm 0.11$

$0.57 \pm 0.13$

\begin{tabular}{|c|c|c|c|c|c|c|}
\hline Total Acids and esters (54) & $35.68 \pm 0.74$ & $5.51 \pm 0.09$ & $8.89 \pm 0.62$ & $27.36 \pm 1.92$ & $66.53 \pm 2.33$ & $53.83 \pm 2.95$ \\
\hline \multicolumn{7}{|l|}{ Pyrazines } \\
\hline 2,5-Dimethyl-pyrazine & - & - & $2.99 \pm 0.13$ & $0.15 \pm 0.01$ & $0.10 \pm 0.00$ & $0.28 \pm 0.01$ \\
\hline $\begin{array}{l}\text { 2,5-Dimethyl-3-(3-methylbutyl)- } \\
\text { pyrazine }\end{array}$ & - & - & $6.60 \pm 0.23$ & $0.78 \pm 0.13$ & $0.27 \pm 0.01$ & $0.87 \pm 0.00$ \\
\hline 2-Butyl-3,5-dimethyl-pyrazine & - & - & $15.24 \pm 1.02$ & $1.78 \pm 0.40$ & $0.54 \pm 0.03$ & $0.46 \pm 0.00$ \\
\hline 3-Ethyl-2,5-dimethyl-pyrazine & - & - & $0.33 \pm 0.01$ & $0.16 \pm 0.06$ & $0.27 \pm 0.00$ & $0.15 \pm 0.01$ \\
\hline 3,5-Diethyl-2-methyl-pyrazine & - & - & $0.51 \pm 0.40$ & - & - & $1.33 \pm 0.05$ \\
\hline Tetramethyl-pyrazine & - & - & $4.81 \pm 0.25$ & $0.84 \pm 0.21$ & $0.17 \pm 0.02$ & $0.44 \pm 0.03$ \\
\hline Total Pyrazines (6) & $0.00 \pm 0.00$ & $0.00 \pm 0.00$ & $30.48 \pm 2.04$ & $3.71 \pm 0.45$ & $1.35 \pm 0.06$ & $3.53 \pm 0.10$ \\
\hline \multicolumn{7}{|l|}{ Furans } \\
\hline 2-Pentyl-furan & $0.14 \pm 0.04$ & $2.16 \pm 0.04$ & $0.27 \pm 0.02$ & $0.56 \pm 0.08$ & $0.32 \pm 0.03$ & $0.86 \pm 0.07$ \\
\hline
\end{tabular}


Table 1. (Continue)

\begin{tabular}{lcccccc}
\hline Volatile compounds & CNF1 & CNF2 & TNM & TNB & TNR & TNBR \\
\hline 2,3-Dihydro-benzofuran & $1.18 \pm 0.04$ & $2.30 \pm 0.70$ & $1.10 \pm 0.11$ & $2.66 \pm 0.75$ & $1.57 \pm 0.10$ & $0.76 \pm 0.16$ \\
\hline Total Furans (2) & $1.32 \pm 0.08$ & $4.46 \pm 0.74$ & $1.37 \pm 0.13$ & $3.22 \pm 0.83$ & $1.89 \pm 0.13$ & $1.62 \pm 0.23$ \\
\hline Sulphur-containing compounds & & & & &
\end{tabular}

2-nitro-N-phenyl-benzenesulfonamide

$\mathrm{N}, \mathrm{N}$ dimethylmethanesulfonamide

Di-tert-dodecyl disulfide

$0.24 \pm 0.07$

$0.30 \pm 0.03$

1-Propenyl-sulfide

$\begin{array}{lll}- & - & 0.35 \pm 0.02\end{array}$

\section{Total Sulphur-containing compounds}

$0.41 \pm 0.00$

$0.00 \pm 0.0$

$0.35 \pm 0.02$

$0.68 \pm 0.01$

$0.54 \pm 0.02$

$1.15 \pm 0.00$

$0.93 \pm 0.07$

$17.11 \pm 0.85$

$2.90 \pm 0.03$

$0.15 \pm 0.04$

$1.76 \pm 0.11$

$10.76 \pm 0.05$

2,6-Dimethoxy-phenol

2-Methoxy-4-(2-propenyl)phenol

3-Phenyl-pyridine

2-Phenyl-pyridine

2,4-Di-tert-butylphenol

2,6-Dimethoxy-phenol

2,6-Di-tert-butylphenol

Butylated Hydroxytoluene (BTH)

2,3,5 Trimethyl phenol

2,3,6 Trimethyl phenol

1,1-Dimethyl-cyclopropane

Octamethyl-cyclotetrasiloxane

Decamethyl-cyclopentasiloxane

1-Undecene

$1.02 \pm 0.02$

1-Pentadecene

4-ethenyl-4-methyl-3- (1-

methylethenyl)-1-(1-methylethyl )-, (3R

$0.43 \pm 0.04$

$0.78 \pm 0.01$

Muurolene

Naphthalene, 1,2,3,4-tetrahydro-1, 6dimethyl-4-(1-methylethyl)-, (1S -cis)1H-Cycloprop[e] azulene, 1a,2,3,4,4a,5,6,7b-octahydro-

1,1,4,7-tetramethyl-, [1aR-

(1a.alpha.,4.alpha.,4a .beta.,7b.alpha.)]

Tetradecane

$0.78 \pm 0.86$

$$
\begin{aligned}
& 1.42 \pm 0.10 \\
& 0.47 \pm 0.01
\end{aligned}
$$

1-Tridecene

Dodecamethyl-pentasiloxane

trans-Calamenene

$1.36 \pm 0.64$

2,3,5,6-Tetrafluoroanisole

3-Methyl-4-phenyl-1H-pyrrole

N-(1-methyl-3-phenylpropyl) acetamide

1,2-Dipropyl-hydrazine

3,4-Dihydroxyphenylglycol

$0.13 \pm 0.01$

$1.15 \pm 0.00$

$0.38 \pm 0.00$

$0.15 \pm 0.03$

$0.36 \pm 0.00$

$0.15 \pm 0.01$

$0.28 \pm 0.12$

$0.11 \pm 0.01$

$0.20 \pm 0.02$

$0.56 \pm 0.09$

$0.52 \pm 0.06$

$0.37 \pm 0.00$

$\begin{array}{cccc}0.23 \pm 0.04 & 0.44 \pm 0.03 & 0.19 \pm 0.04 & 0.16 \pm 0.00 \\ - & - & - & 0.15 \pm 0.01 \\ - & 0.15 \pm 0.02 & 0.20 \pm 0.04 & 0.47 \pm 0.02 \\ - & 0.29 \pm 0.05 & - & 0.13 \pm 0.04 \\ - & - & - & 0.06 \pm 0.00 \\ - & - & - & 0.06 \pm 0.00 \\ - & - & - & 0.06 \pm 0.00 \\ - & - & - & 0.06 \pm 0.00 \\ - & 0.45 \pm 0.06 & - & 0.10 \pm 0.00\end{array}$

$0.14 \pm 0.01$

$0.25 \pm 0.01$

$0.31 \pm 0.37$

$0.15 \pm 0.00$

$0.36 \pm 0.06$

$0.10 \pm 0.00$

$0.22 \pm 0.02$

$0.39 \pm 0.01$

$0.14 \pm 0.14$

$0.18 \pm 0.08$

$0.11 \pm 0.07$

$0.08 \pm 0.00$

$2.74 \pm 0.71$

$0.11 \pm 0.00$

\section{$0.19 \pm 0.02$}

$0.28 \pm 0.10$

$-$

-
-
-
-

$37 \pm 0.00$
$16 \pm 0.00$
$15 \pm 0.01$
$47 \pm 0.02$
$13 \pm 0.04$
$06 \pm 0.00$
$06 \pm 0.00$
$06 \pm 0.00$
$06 \pm 0.00$
$10 \pm 0.00$


Table 1. (Continue)

\begin{tabular}{|c|c|c|c|c|c|c|}
\hline Volatile compounds & CNF1 & CNF2 & TNM & TNB & TNR & TNBR \\
\hline $\begin{array}{l}\text { 1,3,5,7-Tetraethyl-1-ethylbutoxysi } \\
\text { loxycyclotetrasiloxane }\end{array}$ & - & - & - & - & - & $0.66 \pm 0.01$ \\
\hline Pyroquilon & - & - & - & - & - & $0.26 \pm 0.00$ \\
\hline Dodecamethyl-cyclohexasiloxane & - & - & - & - & $0.31 \pm 0.01$ & $0.04 \pm 0.01$ \\
\hline 1,2-Di-but-2-enyl-cyclohexane & - & - & - & - & - & $0.07 \pm 0.02$ \\
\hline $\begin{array}{l}\text { 4-[3-(4 Chlorobenzyloxy)propyl]-1H- } \\
\text { imidazole }\end{array}$ & - & - & - & - & $0.08 \pm 0.01$ & $0.05 \pm 0.00$ \\
\hline $\begin{array}{l}\text { Estra-1,3,5(10)-trien-17-one, } 2 \\
{[(\text { trimethylsilyl) amino }]-3 \text { - }}\end{array}$ & - & - & - & - & - & $0.24 \pm 0.01$ \\
\hline Total Miscellaneous ( 37 ) & $5.60 \pm 1.11$ & $33.44 \pm 1.93$ & $7.47 \pm 0.39$ & $20.12 \pm 1.68$ & $7.47 \pm 0.85$ & $10.28 \pm 2.20$ \\
\hline
\end{tabular}

Values are means \pm standard deviation $(\mathrm{n}=2$ )

- = Not detectable

CNF1 = boiled non-fermented soybean

CNF2 $=$ autoclaved non-fermented soybean

$\mathrm{TNM}=$ fermented by natural microorganisms

TNB $=$ fermented with pure $B$. subtilis TN51

$\mathrm{TNR}=$ fermented with pure $R$. oligosporus

$\mathrm{TNBR}=$ fermented with co-pure $B$. subtilis TN51 and R. oligosporus.

(cooked by boiling) could be considered as "original" (or initial) for the soybean raw material of the TNM, prior to fermentation, whereas the CNF2 (cooked by autoclaving) could be for the TNB, TNR, and TNBR.

For the CNF samples, it was found that total volatiles found in the autoclaved soybeans (CNF2) were higher than those in the boiled soybeans (CNF1). In total, 27 and 24 volatile components were identified from the CNF1 and CNF2, respectively. They belonged to 7 classes of compounds including alcohols (3 for $\mathrm{CNF} 1$, and 4 for CNF2), aldehydes (3 and 4), ketones (4 and 1), acids and esters (8 and 4), furans (2 and 2), sulphur-containing (1 and 0$)$, and miscellaneous compounds (6 and 9). The CNF2 samples also had higher concentration levels of the volatile compounds in the groups of alcohols (5.34 times), aldehydes (9.22 times), furans (2.61 times), and miscellaneous (4.61 times), when compared with the CNF1 sample. However, the CNF2 sample had lower concentrations in the groups of ketones (19.38 times), acids and esters (8.40 times), and sulphur-containing compounds. This finding indicates that different cooking procedure (i.e., boiling and autoclaving) can cause a change in the volatile components. This is in agreement with Dakwa et al. (2005) and Dajanta et al. (2011) reporting that alcohols and aldehydes were more abundant in the nonfermented soybeans cooked by high temperature, in which they suggested that the abundance of these compounds possibly occurred by the Maillard reaction.
When using the microbial inoculum, however, it clearly showed that the fermentation process was able to induce an increase in both amounts and types of the volatile components. For instance, there were more volatile compounds in the TNM sample, when compared to those of the CNF1 considered as an "original" substrate. Similarly, the same finding was also found in the TNB, TNR, and TNBR samples, when compared their volatiles with those of the CNF2 counterparts. Interestingly, there were much volatiles which could be identified only in the fermented soybean products; these included 14 alcohols, 9 aldehydes, 8 aromatics, 15 ketones, 43 acids and esters, 6 pyrazines, 3 sulphurcontaining, and 26 miscellaneous (Table 1). It can be therefore concluded that these volatile components were bio-synthesised via microbial transformation.

The volatile compounds detected from the fermented soybean products were abundant. In total, there were 44 , 67,73 , and 102 volatiles that could be identified from the TNM, TNB, TNR, and TNBR, respectively. Their amounts also increased ranging from $4.80-59.78 \%$. For detailed analysis, each volatile class was compared as follows.

Alcohols: There were 20 alcohols which could be detected from the samples. Of these, the CNF2 exhibited highest concentration levels; this was mainly due to the very high amounts of 1-octen-3-ol. This compound, present in all soybean samples, is the key compound 
yielding a beany flavor (Arai et al., 1966). Fourteen alcohols were distinct to the fermented products and the most diverse levels of alcohols were found in the TNBR (Table 1). It is suggested that the alcohol compounds might have been liberated from enzymatic lipid oxidation of the unsaturated fatty acids (Kato et al., 1981).

Aldehydes: Fifteen aldehydes were identified from the soybean samples; of these, nine could be detected only from the fermented samples. High levels of aldehydes were found in the CNF2 and TNM samples. Some aldehydes such as benzaldehyde and benzene acetaldehyde considered as having a favourable odour (Chung, 2000) were present in most samples (Table 1). The aldehydes can occur via lipid oxidation and degradation (Ames and Macleod, 1984).

Ketones: In this study, a total of 19 ketones were detected from the soybean samples. Fifteen of these were identified only from the fermented samples. The ketone class in the TNM sample significantly decreased from its origin (CNF1) whereas there was an increase of the ketone group in the TNB, TNR, and TNBR samples (compared to the CNF2). This finding is different from Dajanta et al. (2011) reporting that the ketone class was more abundant when using the naturally occurring microbes in the fermentation process. The common ketone was acetoin. Ketones are in general derived from an amino acid and lipid degradation and the presence of these compounds has a high impact on food odour (Owens et al., 1997).

Acids and Esters: For the acids and esters class, there were 54 components of which 43 were unique to the fermented soybean products. The TNM sample showed four times fewer contents than the CNF1. In contrast, the concentrations of this group increased dramatically (ranging from $5-12$ times) in the TNB, TNR, and TNBR samples. Organic acids were mainly found in the CNF1 (i.e., octanoic and octadecanoic acids), and were minor in the CNF2 as well as the fermented samples. Some acids including 2methylbutanoic acid and 2-methylpropanoic acid, have been synthesized through leucine and isoleucine metabolism as suggested by Larroche et al. (1999). In the present study, many alkyl esters were identified especially for the fermented soybean products. The presence of the ethyl esters, in particular, has been reported as a "typical" flavor compounds of the fermented soybeans including Chungkukjang and Doenjang (Tanaka et al., 1998; Chung, 1999).

Pyrazines: One of the most characteristic groups of the components in these fermented soybeans was pyrazines. As shown in Table 1, pyrazines were absent from the cooked, non-fermented soybeans (CNF1 and CNF2), and subsequently appeared only after the fermentation. Pyrazines were much more abundant in the TNM than other fermented soybeans. Interestingly, this finding is in accordance with Dajanta et al. (2011) work and thus further confirms an importance of the naturally occurring microbes in pyrazine production. Pyrazine class is unique and has been reported to contribute to the roasted feature of the food products (Maga 1992). In addition, pyrazines are also commonly found in the fermented soybean products (Sugawara et al., 1998; Leejeerajumnean et al., 2001; Dajanta et al., 2011). The presence of these pyrazines is related to sensory attributes as a flavourable odour (i.e., nutty and roasted odour) (Lee and Ahn, 2009). Although the pyrazines can be formed via the thermal reactions, it is obvious that the occurrence of the pyrazines detected in this study was by microbial transformation. Besson et al. (1997) described a biosynthesis of 2,5-dimethylpyrazine and tetramethylpyrazine by using Bacillus subtilis.

Others: Aromatic, furan, and sulphur-containing compounds were identified and found as a minor group (less than $5 \%$ of total volatile compounds). Aromatics and sulphur-containing compounds were mainly found in the fermented soybean products. It should be noted, however, that sulphur-containing compounds have a great impact on the product odour, albeit their low concentration levels. The presence of these substances (i.e., dimethyl disulphide) gives rise to sulphur and fishy odour (Lo et al., 2008). Furans, on the other hand, were commonly found in all samples. Only two derivatives (2pentyl-furan and 2,3-dihydro-benzofuran) were identified in which their concentrations ranged from 1.32 $-4.46 \%$.

\section{Conclusion}

In conclusion, a total of 165 volatiles could be detected in the soybean and the fermented soybean samples used in the present study. There was a shift among the volatile classes depending on the cooking process and the microbes used for fermentation. The data obtained here are expected to provide qualitative and quantitative information regarding the distribution and development of the volatile components during the soybean fermentation. In terms of consumer's acceptability, further study should be undertaken to identify the important odorous compounds which might be correlated to taste, flavour, and nutrition. 


\section{Acknowledgements}

The authors gratefully acknowledge the Asia Research Centre, Chulalongkorn University (no. 005/2559), and Mae Fah Luang University for their financial support.

\section{References}

Ames, J.M. and Macleod, G. (1984). Volatile Components of an Unflavored Textured Soy Protein. Journal of Food Science, 49, 1552-1565.

Arai, S., Suzuki, H., Fujimaki, M. and Sakurai, Y. (1966). Studies on Flavor Components in Soybean: Part II. Phenolic Acids in Defatted Soybean Flour. Agricultural and Biological Chemistry, 30, 364-369.

Besson, I., Creuly, C., Gros, J.B. and Larroche, C. (1997). Pyrazine production by Bacillus subtilis in solid-state fermentation on soybeans. Applied Microbiology and Biotechnology, 47, 489-495.

Chung, H.Y. (1999). Volatile components in fermented soybean (Glycine max) curds. Journal of Agricultural and Food Chemistry, 47, 2690-2696.

Chung, H.Y. (2000). Volatile flavor components in red fermented soybean (Glycine max) curds. Journal of Agricultural and Food Chemistry, 48, 1803-1809.

Dajanta, K., Wongkham, S., Thirach, P., Baophoeng, P., Apichartsrangkoon, A., Santithum, P. and Chukeatirote, E. (2009). Comparative study of proteolytic activity of protease-producing bacteria isolated from thua nao. Maejo International Journal of Science and Technology, 3, 269-276.

Dajanta, K., Apichartsrangkoon, A. and Chukeatirote, E. (2011). Volatile profiles of thua nao, a Thai fermented soy product. Food Chemistry, 125, 464470.

Dakwa, S., Sakyi-Dawson, E., Diako, C., Annan, N.T. and Amoa-Awua, W.K. (2005). Effect of boiling and roasting on the fermentation of soybeans into dawadawa (soy-dawadawa). International Journal of Food Microbiology, 104, 69-82.

Hui, Y. H. and Evranuz, E.Ö. (2012). Handbook of plant -based fermented food and beverage technology. $2^{\text {nd }}$ ed. Boca Raton, FL: CRC Press.

Kato, H., Doi, Y., Tsugita, T., Kosai, K., Kamiya, T. and Kurata, T. (1981). Changes in volatile flavour components of soybeans during roasting. Food Chemistry, 7, 87-94.

Kiuchi, K. and Watanabe, S. (2004). Industrialization of Japanese natto. In Steinkraus, K.H. (Ed.),
Industrialization of indigenous fermented foods. $2^{\text {nd }}$ ed., p. 193-246. New York: Marcel Dekker,

Larroche, C., Besson, I. and Gros, J.B. (1999). High pyrazine production by Bacillus subtilis in solid substrate fermentation on ground soybeans. Process Biochemistry, 34, 667-674.

Lee, S. and Ahn, B. (2009). Comparison of volatile components in fermented soybean pastes using simultaneous distillation and extraction (SDE) with sensory characterisation. Food Chemistry, 114, 600609.

Leejeerajumnean, A., Duckham, S.C., Owens, J.D. and Ames, J.M. (2001). Volatile compounds in Bacillusfermented soybeans. Journal of the Science of Food and Agriculture, 81, 525-529.

Lo, Y.C.M., Koziel, J.A., Cai, L., Hoff, S.J., Jenks, W.S. and Xin, H. (2008). Simultaneous chemical and sensory characterization of volatile organic compounds and semi-volatile organic compounds emitted from swine manure using solid phase microextraction and multidimensional gas chromatography-mass spectrometry-olfactometry. Journal of Environmental Quality, 37, 521-534.

Maga, J.A. (1992). Pyrazine update. Food Reviews International, 8, 479-558.

Owens, J.D., Allagheny, N., Kipping, G. and Ames, J.M. (1997). Formation of volatile compounds during Bacillus subtilis fermentation of soya beans. Journal of the Science of Food and Agriculture, 74, 132-140.

Qin, L. and Ding, X. (2007). Formation of taste and odour compounds during preparation of Douchiba, a Chinese traditional soy-fermented appetizer. Journal of Food Biochemistry, 31, 230-251.

Steinkraus, K.H. (2002). Fermentations in world food processing. Comprehensive Reviews in Food Science and Food Safety, 1, 23-32.

Sugawara, E., Suzuki, T. and Yoshida, Y. (1998). The comparison of pyrazine compounds in non-salted fermented soybean products. Food Science and Technology International, 4, 85-88.

Tanaka, T., Muramatsu, K., Kim, H., Watanabe, T., Takeyasu, M., Kanai, Y. and Kiuchi, K. (1998). Comparison of volatile compounds from ChungkukJang and Itohiki-Natto. Bioscience, Biotechnology, and Biochemistry, 62, 1440-1444. 